\title{
OAO-2 OBSERVATIONS OF THE NEAR ULTRAVIOLET EXTINCTION FEATURE
}

\author{
BLAIR D. SAVAGE \\ Space Astronomy Laboratory, Washburn Observatory, University of Wisconsin, \\ Madison, Wis. U.S.A.
}

\begin{abstract}
OAO-2 near UV spectral scans of 75 early-type stars have been examined for evidence of the UV extinction bump. The bump has been detected in 40 of these stars. The position of the maximum of the bump is within $\pm 40 \AA$ of $2175 \AA$ in all cases. A color excess between the wavelengths $2175 \AA$ and $3500 \AA$ was determined for all 40 stars. This color excess, which is a measure of the bump strength, correlates very well with $E(B-V)$ except for stars associated with nebulosity.
\end{abstract}

In a recent paper Bless and Savage (1972, hereafter referred to as Paper I) presented ultraviolet extinction curves for 17 stars. These curves were derived from Orbiting Astronomical Observatory 2 (OAO-2) data and covered the spectral region $\lambda 1100$ 3600. The most significant feature of these curves was the well defined bump in extinction which peaked at $2175 \AA$. The purpose of the present paper is to briefly discuss new observations of this very important feature.

The solid curve in Figure 1 shows the 'average' ultraviolet extinction curve obtained in Paper I. We have plotted extinction in magnitudes vs $1 / \lambda$ in $\mu^{-1}$. The extinction is normalized to zero at the $\mathrm{V}$ bandpass and to $E(B-V)=1.0$. The curves for $\zeta$ Oph and $\theta^{1+2}$ Ori illustrate the range of variability in extinction found so far. These curves were obtained from an analysis of low resolution $(\Delta \lambda \sim 10-20 \AA)$ spectral scans of reddened early-type stars. OAO-2 contains two objective grating spectral scanners; one covers the near ultraviolet and the other the far ultraviolet. The relationship between this spectral coverage and the ultraviolet extinction is illustrated in Figure 1. As can be seen, Spectrometer 1 covers the region of the ultraviolet extinction bump while Spectrometer 2 covers the minimum and subsequent rise in extinction toward short wavelengths. Unfortunately Spectrometer 1 operated successfully for only $1 \frac{1}{2} \mathrm{yr}$. In comparison, at the time of writing ( $3 \frac{1}{2} \mathrm{yr}$ after launch) Spectrometer 2 continues to operate perfectly. This paper mainly concerns an analysis of all the good Spectrometer 1 scans for normal stars of spectral type B3 or earlier.

Since large variations are seen in the normalized ultraviolet extinction curves it is of interest to investigate the systematics of these variations in a large sample of stars. With this project in mind, we have defined the color excess, $E(2175-3500)$, which is a measure of the extinction between $2175 \AA$ and $3500 \AA .2175 \AA$ is the average position of the maximum in the ultraviolet extinction bump. This color excess is illustrated in Figure 1. One can see that this quantity gives a good measure of the extinction in the near ultraviolet bump. Fortunately, this quantity can be derived from individual near ultraviolet spectrometer scans of reddened stars whereas other measures of the bump, for example the height above the dashed line in Figure 1, require both near and far ultraviolet data. Both sets of data are available for only a limited number of reddened stars. 


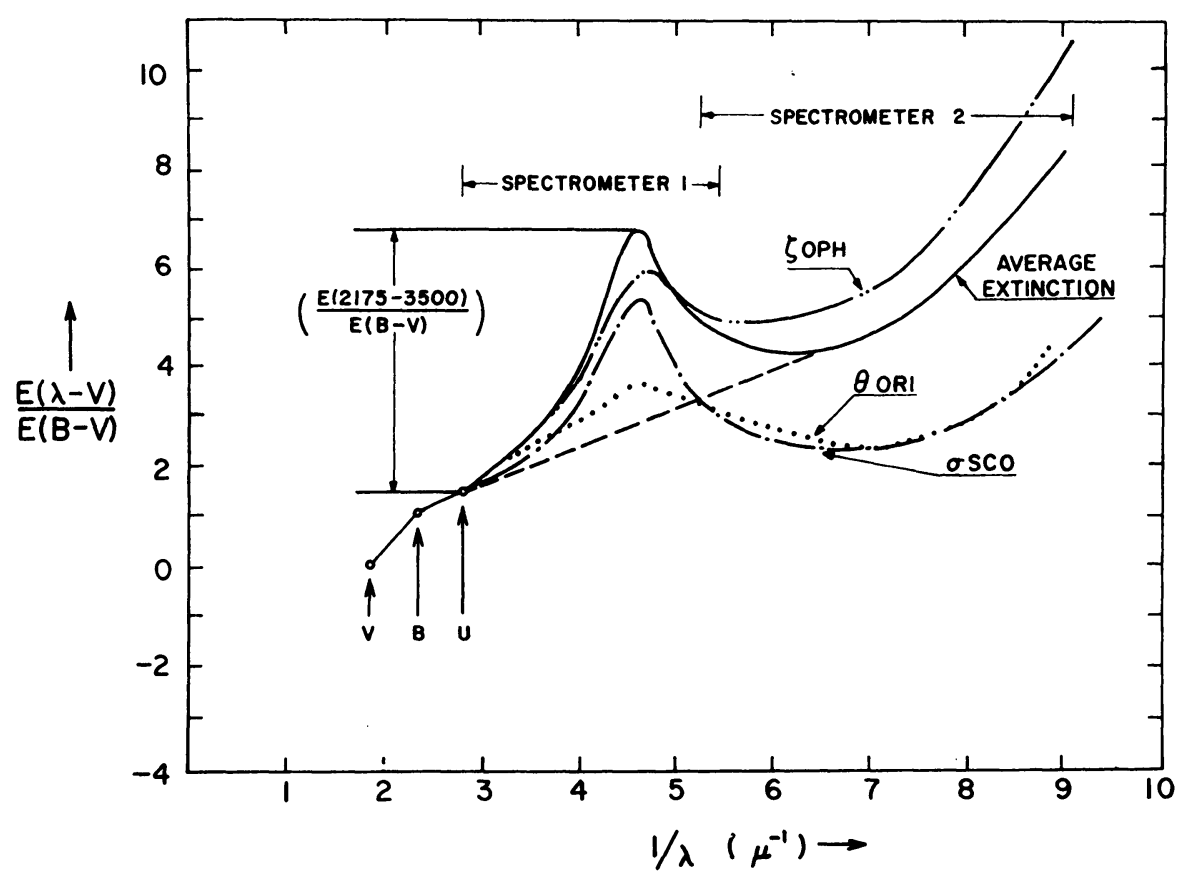

Fig. 1. Ultraviolet extinction curves from Bless and Savage (1972). The curves for $\zeta$ Oph and $\theta^{1+2}$ Ori illustrate the range of variability in ultraviolet extinction found so far. The wavelength coverage of each OAO-2 spectrometer is indicated above the extinction curves. $E(2175-3500)$ refers to the color excess which gives a measure of the amount of additional extinction in the near ultraviolet bump.

In order to derive an extinction curve or a color excess one must of course know the intrinsic behavior of stars of different spectral type and luminosity class. A detailed discussion of this subject was given in Paper I. The basic result of that analysis was that the intrinsic ultraviolet energy distributions of normal early-type stars correlates well with $(B-V)_{0}$, the intrinsic $(B-V)$ color. Furthermore this result does not depend sensitively on luminosity class. Emission line stars often deviate from normal stars due to the presence of continuous Balmer emission in the near ultraviolet. Except where noted stars with Be characteristics have been omitted from this program. In this paper we have continued the investigation of intrinsic properties of stars. Figure 2 shows a color-color plot for normal early-type stars with $E(B-V) \leq 0.10$. The ultraviolet color spans the wavelengths 2175 and $3500 \AA$ and was obtained from near ultraviolet scanner data. The colors plotted have been corrected to intrinsic colors by application of the 'average' extinction curve shown in Figure 1. One can see that the near ultraviolet color correlates well with $(B-V)_{0}$ and there are no pronounced luminosity class effects. $95 \%$ of the stars are contained in a band $\pm 0{ }^{m} \cdot 15$ from the dashed line (a visual estimate of the mean relation). We can conclude that a star's $(B-V)_{0}$ is a good measure of its intrinsic near ultraviolet energy distribution. The amount of scatter in Figure $2( \pm 0.15)$ can be considered to be a conservative estimate of the uncertainty in measures of $E(2175-3500)$ to be discussed shortly. As will be seen this 


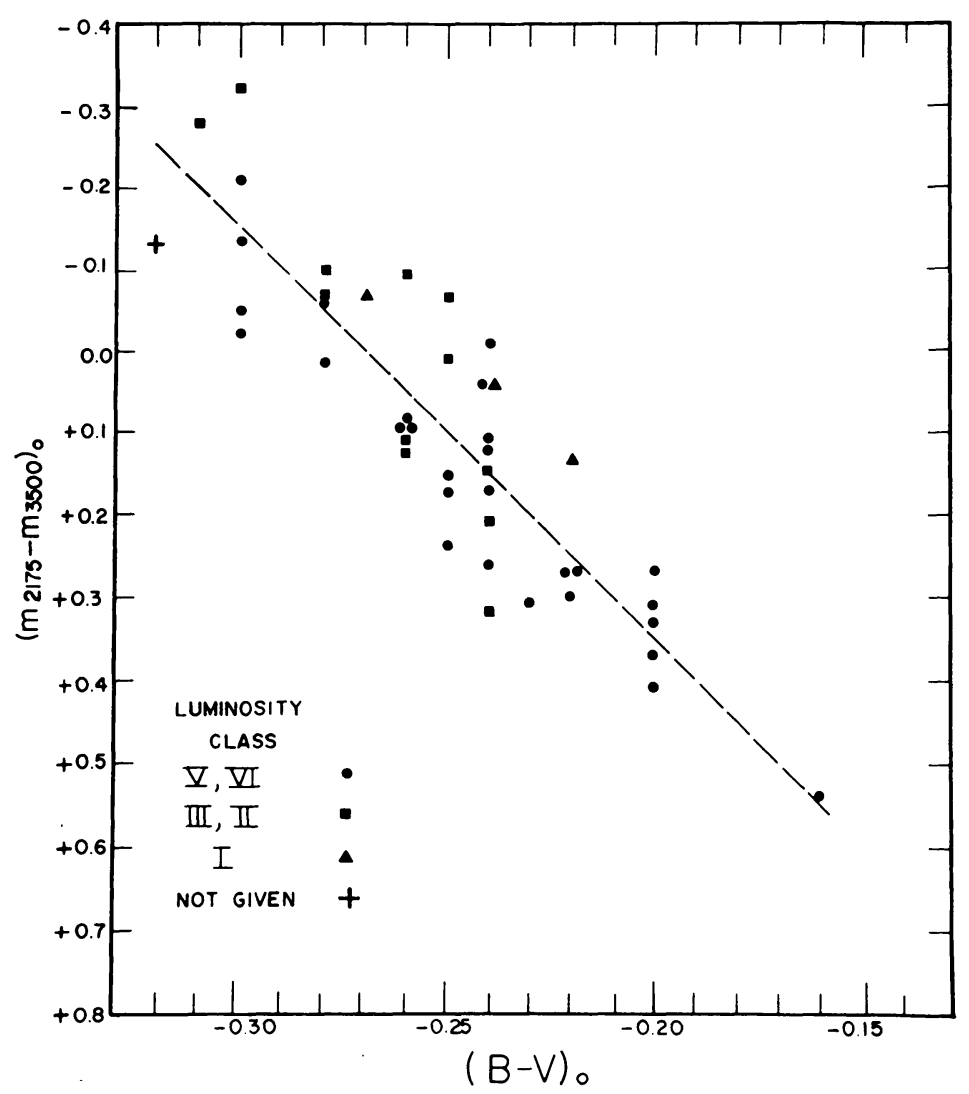

Fig. 2. A color-color plot obtained from OAO-2 Spectrometer 1 scans of slightly reddened stars $(E(B-V)<0.10) . m_{2175}-m_{3500}$ refers to a color that spans the wavelengths 2175 and $3500 \AA$. The colors plotted have been corrected to zero reddening by using the average extinction curve seen in Figure 1. The symbols indicate stars of various luminosity classes. The dashed line represents the mean relation between the near ultraviolet color and $(B-V)_{0}$.

uncertainty is much smaller than the variations of normalized near ultraviolet extinction observed from object to object. Underhill (1972) has suggested that differences in line blanketing between stars with the same visual spectra could introduce large errors into the derived extinction curves, particularly in the vicinity of $2200 \AA$. On the basis of the good correlation in the observed sample of stars seen in Figure 2 and also in Figure 4 of Paper I we must conclude that Underhill's suggestion is not supported by the observations.

During its lifetime, the near ultraviolet scanner on OAO-2 obtained spectral scans of 75 normal stars of spectral type B3 or earlier. We have searched all these scans for evidence of the near ultraviolet extinction feature. The method was simply to intercompare all the spectrometer scans with those of a few very slightly reddened stars $(E(B-V)<0.02)$. By this process the extinction bump was detected in 40 of 75 stars. Those stars with a detectable near ultraviolet feature had $E(B-V)$ in the range 0.04 to 
0.61. The 35 stars in which the feature could not be detected usually had $E(B-V)<$ 0.05 . In all 40 cases the wavelength of the maximum of the bump was within $\pm 40 \AA$ of $2175 \AA$. The spread of $\pm 40 \AA$ probably mostly reflects observational scatter rather than a true variation of the bump position. The stars with detectable extinction bumps range in distance from 150 to $1500 \mathrm{pc}$ and are mostly located in associations confined to regions near the plane of the Galaxy. Many stars are in Scorpius-Ophiuchus (11) and Orion (10) with smaller numbers in Cygnus-Cepheus (8), Perseus (3) and elsewhere (8).

For each of the 40 stars with a detectable near ultraviolet extinction feature the color excess $E(2175-3500)$ was derived by taking the dashed line in Figure 2 to represent the intrinsic near ultraviolet color of stars as a function of $(B-V)_{0}$. Figure 3 shows the correlation between $E(2175-3500)$ and $E(B-V)$; the dashed line is for the relation $E(2175-3500)=5.5 E(B-V)$. The correlation is generally excellent if stars associated with nebulosity are excluded (squares). The error bars plotted in Figure 3 simply represent the conservative estimate of $\pm 00^{m} 15$ obtained from a visual inspection of Figure 2. The good correlation between $E(B-V)$ and $E(2175-3500)$ indicates that the near ultraviolet extinction bump is a persistent feature in the extinction curve

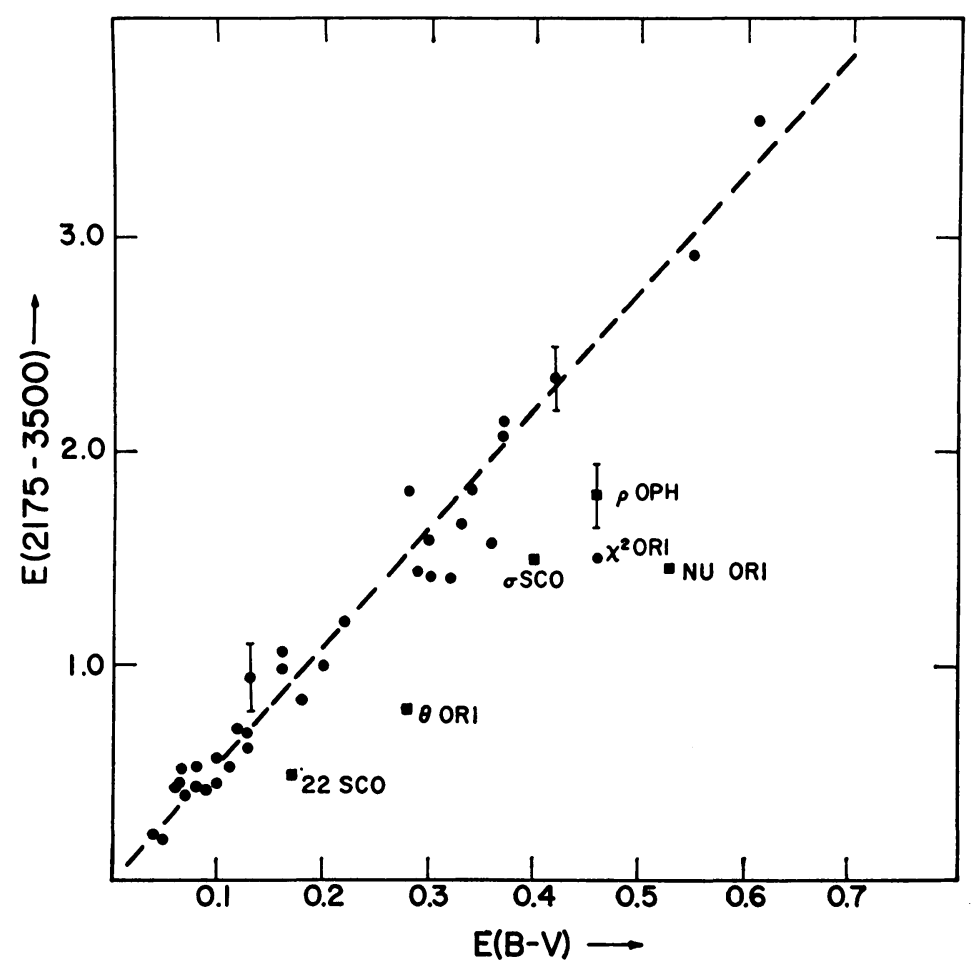

Fig. 3. A correlation between the near ultraviolet color excess and $E(B-V)$ for 40 stars. $E(2175-3500)$ is basically a measure of the excess extinction in the near ultraviolet bump. Stars associated with nebulosity are marked with squares $(\boldsymbol{D})$ and other stars are marked with circles $(\bullet)$. The dashed line represents the relation $E(2175-3500)=5.5 E(B-V)$. 
TABLE I

Stars with abnormal near ultraviolet extinction

\begin{tabular}{|c|c|c|c|}
\hline Object & S.T. & $E(B-V)$ & Comments \\
\hline \multirow[t]{2}{*}{$\theta^{1+2}$} & $06_{p}$ & 0.36 & $\begin{array}{l}\text { Associated with emission nebula. } \\
\text { Visual and infrared extinction abnormal (Lee, 1968). }\end{array}$ \\
\hline & $09.5 \mathrm{~V}_{p}$ & 0.21 & $\begin{array}{l}\theta^{2} \text { Ori exhibits large } P_{V} / P_{B} \text { (Serkowski, 1968) and variable } \\
\text { polarization (Coyne and Gehrels, 1967). The variable polar- } \\
\text { ization indicates circumstellar shell activity and the possi- } \\
\text { bility of contamination from continuous Balmer emission in } \\
\text { the near ultraviolet. The extinction measurement for this } \\
\text { star should be treated with caution. }\end{array}$ \\
\hline NU Ori & B1V & 0.53 & $\begin{array}{l}\text { Associated with nebulosity. } \\
\text { Visual and infrared extinction abnormal (Lee, 1968). } \\
\text { Large } P_{V} / P_{B} \text { (Serkowski, 1968). }\end{array}$ \\
\hline$\chi^{2}$ Ori & B2Ia & 0.46 & $\begin{array}{l}\text { No associated nebulosity. } \\
\text { Variable polarization (Vitrichenko and Efimov, 1965) indicates } \\
\text { shell activity and possibility of contamination by continuous } \\
\text { Balmer emission. The extinction measurement should be } \\
\text { treated with caution. }\end{array}$ \\
\hline$\sigma$ Sco & B11II & 0.40 & Associated with reflection nebula (Hubble, 1922). \\
\hline$\varrho$ Oph & $\begin{array}{l}\text { B2IV } \\
\text { B2V }\end{array}$ & 0.46 & $\begin{array}{l}\text { Associated with reflection nebula (Hubble, 1922). } \\
\text { Large } P_{V} / P_{B} \text { (Serkowski, 1968). }\end{array}$ \\
\hline $22 \mathrm{Sco}$ & $\mathrm{B} 2 \mathrm{~V}$ & 0.17 & Associated with reflection nebula (Hubble, 1922). \\
\hline
\end{tabular}

of stars. Furthermore Figure 3 gives additional support to our contention that Underhill's suggestion about large errors being introduced into extinction measurements by variable ultraviolet line blanketing is wrong.

Six stars deviate significantly from the generally good correlation seen in Figure 3. Table I lists these stars and gives relevant information about their characteristics. A significant fact is that five of these six stars are associated with nebulosity. In addition a few of these stars are known to have peculiar visual and infrared extinction and abnormally large ratios of visual to blue polarization $\left(P_{V} / P_{B}\right)$. As pointed out in Paper I it's possible that the acceptance of both stellar and nebular radiation by the OAO scanners produces the abnormal ultraviolet extinction measurements. In the near ultraviolet the important possibilities for nebular contamination are continuous Balmer and two photon emission from atomic hydrogen, and scattering by local dust into the line of sight. Contamination by two photon and Balmer emission should be negligible for all these objects except $\theta^{1+2}$ Ori because the other nebulae shine mostly by reflected light. Code (1973) has discussed the effect local scattering by dust might have on extinction measurements. For particle albedos similar to those presented by Witt and Lillie (1972) one would expect local scattering if important to make the bump appear stronger than normal. The observations reported here, however, indicate the opposite effect.

For several objects with abnormal near ultraviolet extinction additional data are available which can set some limits on the possibility of nebular contaminations. With a spatial resolution of $2^{\prime}$ on direct far ultraviolet images of $\theta^{1+2}$ Ori Henry and 
Carruthers (1970) found no evidence for nebular radiation and an analysis of the stellar images produced a far ultraviolet extinction determination (Weber et al., 1971) in reasonable agreement with the far ultraviolet portion of the $\theta^{1+2}$ Ori curve presented in Paper I (also shown in Figure 1). From a far ultraviolet spectrum of $\sigma$ Sco with an objective spectrograph Jenkins (private communicction) was able to conclude that the observed far ultraviolet radiation comes from a region having an angular diameter of less than 1'. Furthermore direct photographs of $\sigma$ Sco in the visual were made with the University of Wisconsin $16^{\prime \prime} f / 19$ telescope. Using Kodak Tri-X film (the only film readily available) the star was first detected with an exposure of $1 / 50 \mathrm{~s}$. An increase of exposure to $60 \mathrm{~s}$ (a factor of 3000 increase neglecting reciprocity failure) failed to reveal nebulosity. In addition photometry observations of $\sigma$ Sco plus nebulosity in a $40 \AA$ band centered at $\sim 4500 \AA$ with diaphragms of $2^{\prime}$ and $1^{\prime}$ were essentially identical to an accuracy of about $1 \%$. From these data one can conclude that nebular contamination for these objects is probably unimportant unless it arises from a region very close to the stars.

The abnormal extinction for stars associated with nebulosity is most likely due to the local modification of the grains by the intense stellar radiation fields of the illuminating stars. Preferential destruction or ejection of small particles could explain the low near ultraviolet extinction evident in Figure 3. Paper I presented complete extinction curves for four of the six objects having peculiar near ultraviolet extinction. Figure 1 shows two of those curves which deserve additional comment. The near ultraviolet bump is much less pronounced in $\theta^{1+2}$ Ori compared to $\sigma$ Sco. It appears in the case of $\sigma$ Sco that the component mainly responsible for the far ultraviolet extinction rise has been destroyed whereas for $\theta^{1+2}$ Ori both the far ultraviolet component and the component responsible for the near ultraviolet bump have been destroyed. The absence of a strong extinction bump in $\theta^{1+2}$ Ori implies that one should be cautious in interpreting determinations of particle albedos from measurements of reflection nebulae. The results found here and in Paper I strongly suggest that particles which can exist in the general interstellar medium may be quite different from particles which can exist in the vicinity of hot stars.

The star $\chi^{2}$ Ori is the only object listed in Table I that is not associated with nebulosity. This star does, however, exhibit variable polarization (Vitrichenko and Efimov, 1965) which indicates circumstellar shell activity and the possibility that the near ultraviolet data are contaminated by continuous Balmer emission from this shell. The extinction measurement for this star should therefore be treated with caution. A similar criticism applies to $\theta^{2}$ Ori for which Coyne and Gehrels (1967) also detected variable polarization.

As is evident in Table $I$ there appears to be a close relation between stars with peculiar ultraviolet extinction and peculiar polarization in the visual. This characteristic also holds for HD 37903 which has a very large $P_{V} / P_{B}($ Serkowski, 1968) and a peculiar far ultraviolet extinction (Weber et al., 1971). Furthermore HD 37903 is associated with nebulosity.

A number of other correlations can be explored with the new near ultraviolet color 
excess. For example, one very interesting problem is that of finding the carriers of the diffuse interstellar features. Wu (1972) has investigated the strength of the diffuse features at $5780 \AA$ and $4430 \AA$ in stars with measured near ultraviolet excesses and has found that the correlation of the diffuse features with $E(2175-3500)$ shows as much scatter as the correlation of these features with $E(B-V)$. Therefore there is no convincing evidence to indicate that the diffuse features are carried by the material producing the near ultraviolet extinction bump. A possible difficulty with this conclusion is that $E(2175-3500)$ is significantly influenced by the behavior of the extinction curve in the far ultraviolet. This effect is evident in Figure 1 by comparing the extinction curve of $\zeta$ Oph with that of $\sigma$ Sco. Although $\zeta$ Oph has a larger $E(2175$ $3500) / E(B-V)$ than $\sigma \mathrm{Sco}$, the bump is more pronounced in the extinction curve of $\sigma$ Sco. For the purpose of investigating the relationship between diffuse interstellar features and the near ultraviolet bump alternate measures of the bump strength are desirable.

\section{References}

Bless, R. C. and Savage, B. D.: 1972, Astrophys. J. 171, 293.

Code, A. D,: 1973, this volume, p. 505.

Coyne, G. V. and Gehrels, T.: 1967, Astron. J. 72, 887.

Henry, R. C. and Carruthers, G. R.: 1970, Science 170, 527.

Hubble, E.: 1922, Astrophys. J. 56, 416.

Lee, T. A.: 1968, Astrophys. J. 152, 913.

Serkowski, K.: 1968, Astrophys. J. 154, 115.

Underhill, A.: 1972, in A. D. Code (ed.), Proceedings of OAO Symposium, NASA Publication SP-310.

Vitrichenko, E. A. and Efimov, Y. S.: 1965, Izv. Krymsk. Astrofiz. Obs. 34, 114.

Weber, S. V., Henry, R. C., and Carruthers, G. R.: 1970, Astrophys. J. 166, 543.

Witt, A. and Lillie, C.: 1972, in A. D. Code (ed.), Proceedings of O AO Symposium, NASA Publication SP.310.

Wu, C. C.: 1972, Astrophys. J. 178, 681. 